\title{
Prevention of cardiovascular disease
}

\section{[gw22-e0141] EFFECT OF PREVENTIVE HEALTH INTERVENTION PROGRAM ON HYPERTENSION FROM COMMUNITIES IN SHENZHEN CITY}

Yao Gongyuan', Pan Jianqing'2 Li Shuangfei' 'Shenzhen Futian No.2 People' Hospital, Guangdong, China; ${ }^{2}$ Shenzhen Futian Chronic Diseases Control Hospital, Shenzhen, China

10.1136/heartjnl-2011-300867.293

Objectives To assess the effect of preventive health intervention program including health education, lifestyle and behaviour intervention and drug treatment, on hypertension awareness rate, treatment rate and control rate of patients from communities in Shenzhen City.

Methods Thousand and fifty two patients from five Community Health Service Centres in Shenzhen City were enrolled for preventive intervention program from 2008. Intervention contained health education and promotion, behavioural counselling lifestyle intervention and drug treatment. Health education and promotion included distributing handbook about harm, prevention and cure of hypertension and behavioural counselling. Behavioural counselling lifestyle intervention included stopping smoking, restricting alcohol, stopping excessive drinking, low fat, high vitamin, high fibre and low salt diet, keeping broad-minded and optimistic, no less than five times of exercise in 1 week, no less than $1 \mathrm{~h}$ a day and so on. Controlled release drugs were recommended to increase compliance and stable control of blood pressure. Patients' family members were requested to supervise drug treatment. Patients were followed up before the preventive health intervention and at 3 months, 6 months, 12 months, 18 months and 24 months later to assess change of patients' awareness rate, treatment rate and control rate of hypertension and smoking rate, excessive drinking rate, excessive salt intake rate, usually exercise rate.

Results After 2 years follow-up, awareness rate in the 1052 patients rose from $38.87 \%$ to $85.17 \%(=478.6, \mathrm{p}<0.001)$; treatment rate rose from $25.09 \%$ to $66.25 \%(=359.1, \mathrm{p}<0.001)$; control rate rose from $10.17 \%$ to $30.13 \%$ ( $=130.3, p<0.001)$. Excessive drinking rate dropped from $12.55 \%$ to $1.05 \%$ (=109.9, $p<0.001)$; low fat rate dropped from $65.87 \%$ to $36.79 \%(=178.1$, $\mathrm{p}<0.001)$. While smoking rate, excessive salt intake rate and usually exercise rate had no significant changes.

Conclusions Preventive health intervention programs and drug treatment may be the preferred and economical way to prevent and management of hypertension by raising awareness rate, treatment rate, control rate and reducing excessive drinking rate and low fat rate of hypertension patients from communities. 